Article

\title{
Polymer-Based Membranes for Oily Wastewater Remediation
}

\author{
Djamila Zioui ${ }^{1,+}$, Hugo Salazar ${ }^{2,3,+}$, Lamine Aoudjit ${ }^{1,4}$, Pedro M. Martins ${ }^{2,3, * \mathbb{D}}$ and
} Senentxu Lanceros-Méndez 2,5,6,*

1 Unité de Développement des équipements Solaires, UDES/Centre de Développement des Energies Renouvelables, CDER, Bou Ismail, W. Tipaza 42415, Algerie; ziouidjamila@yahoo.fr (D.Z.);

lamineaoudjit@yahoo.fr (L.A.)

2 Centre/Departament of Physics, University of Minho, Campus de Gualtar, 4710-057 Braga, Portugal;

hsalazar@fisica.uminho.pt

3 Institute of Science and Innovation on Bio-Sustainability (IB-S), University of Minho, 4710-057 Braga, Portugal

4 Laboratoire de Chimie du Gaz Naturel, Faculté de Chimie, BP 32, El Alia, U.S.T.H.B., Bab Ezzouar 16111, Algerie

5 BCMaterials, Basque Centre for Materials, Applications and Nanostructures, UPV/EHU Science Park, 48940 Leioa, Spain

6 IKERBASQUE, Basque Foundation for Science, 48013 Bilbao, Spain

* Correspondence: pamartins@fisica.uminho.pt (P.M.M.); senentxu.lanceros@bcmaterials.net (S.L.-M.)

+ These authors contributed equally to this article.

Received: 26 September 2019; Accepted: 21 December 2019; Published: 26 December 2019

\begin{abstract}
The compounds found in industrial wastewater typically show high toxicity, and in this way, they have become a primary environmental concern. Several techniques have been applied in industrial effluent remediation. In spite of the efforts, these techniques are yet to be ineffective to treat oily wastewater before it can be discharged safely to the environment. Membrane technology is an attractive approach to treat oily wastewater. This is dedicated to the immobilisation of $\mathrm{TiO}_{2}$ nanoparticles on poly(vinylidene fluoride-trifluoro ethylene) (PVDF-TrFE) porous matrix by solvent casting. Membranes with interconnected pores with an average diameter of $60 \mu \mathrm{m}$ and a contact angle of $97^{\circ}$, decorated with $\mathrm{TiO}_{2}$ nanoparticles, are obtained. The degradation of oily wastewater demonstrated the high photocatalytic efficiency of the nanocomposite membranes: Under sunlight irradiation for seven hours, colourless water was obtained.
\end{abstract}

Keywords: oil; nanocomposite membranes; PVDF-TrFE; photoreactor; water remediation

\section{Introduction}

The production of oil and gas by petroleum refineries yields a large amount of wastewater, containing mostly organic compounds (aliphatic and aromatic hydrocarbons), inorganic compounds (metals), and dissolved and suspended solids [1].

Nowadays, many techniques are being employed in oily wastewater treatment, such as ultrafiltration [2], ultrasonic separation [3], adsorption [4], and coagulation/flocculation [5], among others. However, these techniques present some drawbacks, such as the large space requirement, low cost and the generation of secondary pollutants. On the other hand, membrane processes are considered a suitable and low-cost alternative to the conventional techniques for treatment of oilfield wastewater, due to unique properties such as the simples process, low energy consumption and no phase transformation [6,7]. Recent studies have reported the use of polymeric membranes for oily wastewater treatment [8-10]. Zhang, B. et al. [11] reported the use of a polytetrafluoroethylene 
(PTFE) membrane for the study of the mechanisms of adsorption fouling of crude oil. Yagoub, $\mathrm{H}$. et al. [12] fabricated a cellulose/chitin/chitosan/PET membrane to be applied in the treatment of oily wastewater. Yang, Y. et al. [13] developed a membrane using palygorskite with a potential application in oil/water separation. A work of Saththasivam, J. et al. [14] reported a new approach for a fast and efficient oil/water separation using a membrane based on $\mathrm{ZnO}$ microspheres and carbon nanotubes. However, membrane fouling has proven to be the major drawback of polymeric membranes use in oily wastewater treatment [11].

Furthermore, the hydrophobicity of polymeric membranes promotes their contamination in the filtration process, especially in oil/water separation, leading to the decrease of water flow and rejection efficiency [15]. Membranes based on PVDF have been investigated for the removal of different pollutants from water, such as copper ions [16], natural organic matter [17], proteins [18], organic compounds [19], volatile organic compounds [20] and desalination [21,22], among others. Of all the PVDF copolymers, poly(vinylidene fluoride-trifluoroethylene) (PVDF-TrFE) presents suitable physicochemical properties for photocatalytic applications, such as a high UV resistance, mechanical and chemical resistance and hydrolytic and thermal stability [23]. This polymer also allows for the production of membranes with controlled porosity and pore size. More recently, a titanium dioxide $\left(\mathrm{TiO}_{2}\right) / \mathrm{PVDF}-\mathrm{TrFE}$ nanocomposite produced by the authors showed a remarkable solar photocatalytic activity in tartrazine degradation - degradation of $78 \%$ under five hours of sunlight irradiation [24].

As a catalyst, titanium dioxide $\left(\mathrm{TiO}_{2}\right)$ is the most widely used photocatalyst for degradation of organic compounds due to its properties, such as non-toxicity, low cost and abundance, physical and chemical stability, superhydrophilicity, and superior photocatalytic activity under UV radiation $(\lambda<390 \mathrm{~nm})$ [25]. The incorporation of $\mathrm{TiO}_{2}$ into polymeric membranes solves some drawbacks related to its use in suspension, namely the recuperation of the nanoparticles.

An efficient alternative to applying photocatalytic membranes are the photocatalytic membrane reactors (PMRs). PMRs have shown a great potential as a "zero" waste process for oily wastewater treatment since they can reduce the loss of catalytic nanoparticles, control the contact time between catalyst and pollutant, and guarantee a continuous process. In this context, PMRs can improve the process efficiency and stability, and reusability of the nanocomposite to reduce the operating cost [26].

Thus, the major focus of this work is to demonstrate $\mathrm{TiO}_{2}$ nanoparticles immobilised on a PVDF-TrFE porous membrane as being an efficient method for the degradation of oil in wastewater in a solar photoreactor, making use of the UV radiation (3-5\%) present in the solar radiation.

\section{Material and Methods}

\subsection{Materials}

PVDF-TrFE (70:30) was obtained from Solvay (Paris, France). $\mathrm{P}_{2} 5^{\circledR}-\mathrm{TiO}_{2}$ nanoparticles were provided by EVONIK (Essen, Germany). Sulfuric acid $\left(\mathrm{H}_{2} \mathrm{SO}_{4}\right) 99 \%$, sodium hydroxide ( $\left.\mathrm{NaOH}\right) 97 \%$, $\mathrm{N}, \mathrm{N}$-dimethylformamide (DMF) 99\%, and methylene (MB) were all purchased from Merck (Darmstadt, Germany).

\subsection{Membrane Preparation}

The solvent casting technique was used to produce the PVDF-TrFE membranes with immobilised

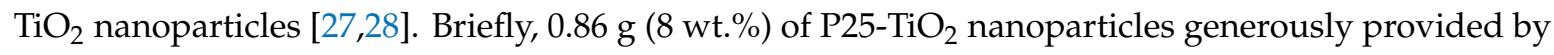
Evonik (Essen, Germany) were added to $90 \mathrm{~mL}$ of $\mathrm{N}, \mathrm{N}$-dimethylformamide (DMF) obtained from Merck (Darmstadt, Germany) and placed in an ultrasound bath for four hours to obtain a homogeneously dispersed solution. The amount of $\mathrm{TiO}_{2}$ used was previously chosen [28] to avoid nanoparticles detachment from the polymer matrix and to preserve its mechanical properties. Afterwards, $10 \mathrm{~g}$ of PVDF-TrFE 70:30 purchased from Arkema (Colombes, France) was added to the solution, achieving a concentration of $10 \mathrm{wt} . \%$ polymer to solvent, and the solution was magnetically stirred until complete polymer dissolution. Ultimately, the solution was placed in glass support, and the solvent evaporated 
at an ambient temperature. When the solvent completely evaporated, a membrane with the same dimensions of the photoreactor surface $(38 \mathrm{~cm}$ length $\times 12 \mathrm{~cm}$ wide) was obtained.

\subsection{Membrane Characterization}

The crystal structure of the $\mathrm{TiO}_{2}$ nanoparticles was evaluated by X-ray diffraction (XRD) using a Bruker D8 Discover diffractometer with incident $\mathrm{Cu} \mathrm{K} \alpha(40 \mathrm{kV}$ and $30 \mathrm{~mA})$. Fourier-transformed infrared spectroscopy (FTIR) was performed to determine the chemical stability of the nanocomposite using an FTIR Alpha instrument (Bruker Corporation, Billerica, MA, USA), over a range of $650-4000 \mathrm{~cm}^{-1}$ using 64 scans with a resolution of $4 \mathrm{~cm}^{-1}$. The scanning electron microscopy (SEM) was obtained with a Quanta 650 SEM (Thermo Fisher, Waltham, MA, USA) to access the morphology/microstructure of the membrane. Energy dispersive X-ray spectroscopy (EDX) was performed with an INCA 350 spectrometer (Oxford Instruments, Abingdon, UK), without gold coating.

The wettability of the samples was measured through contact angle in Physics SCA20 microscope (DataPhysics Instruments GmbH, Filderstat, Germany).

\subsection{Wastewater Sample}

The Hassi R'mel (HR) area is located in southern Algeria, $525 \mathrm{~km}$ south of Algiers, and $120 \mathrm{~km}$ south of Laghouat wilaya, about $70 \mathrm{~km}$ west of Beriane and $120 \mathrm{~km}$ south of Algiers, northwest of Ghardaia. The HR field is centered at $32^{\circ} 55^{\prime} 41^{\circ}$ North and $3^{\circ} 16^{\prime} 16^{\circ}$ East at an average elevation of approximately $750 \mathrm{~m}$ above sea level. The geometric shape, the nature of the effluent and the homogeneity of the reservoir at the HR field led to the selection of three production areas (North, Center, and South) and two compressor stations.

The withdrawal of samples was accomplished on 15 March 2017, at the inlet of the de-oiling unit at CPI S-102 (Corrugated Plate Interceptor), which is a separating basin furnished with a set of parallel plates that facilitate the sedimentation of the non-decanted solid particles. The samples were transported from Hassi R'mel to the Research and Development Center in coolers and stored for $24 \mathrm{~h}$ in a refrigerator at $4^{\circ} \mathrm{C}$ before being used.

\subsection{Oily Wastewater Parameters}

Several parameters were measured before and after the treatment. The temperature (T) was measured using a thermometer ASTM 5C and the electrical conductivity (EC) was measured by a conductivity meter HACH HQ 40d. The suspended solids (SS) were determined by filtering $100 \mathrm{~mL}$ of water to be analyzed through a $47 \mu \mathrm{m}$ pore filter; afterwards, the filter was dried at $105^{\circ} \mathrm{C}$ in an oven for two hours. The amount of suspended solids was estimated by the difference in the weight of the filter before and after drying. The $\mathrm{pH}$ measurements were carried out using a $\mathrm{pH}$-meter type (Inolab PH7310, Weilheim in Oberbayern, Germany). Chemical oxygen demand (COD) was based on the oxidation of organic materials by an excess of potassium dichromate (K2Cr2O7), in acidic medium and boiling, in the presence of silver sulfate and mercury sulfate. COD was measured by a spectrophotometer of the type (DR 1900 LANGE HACH, Duesseldorf, Germany). The percentage of degradation of COD was estimated using the following Equation (1).

$$
\operatorname{Degradation}(\%)=\frac{C_{0}-C_{t}}{C_{0}} \times 100,
$$

where $C_{0}$ and $C_{t}$ are the initial concentration of oxygen and concentration at time $t$, respectively.

The quantification of the total hydrocarbons (HC) in water followed an analytical protocol implemented according to the water standard methods (solvent extraction, purification on florisil cartridge, and finally gas chromatography analysis coupled to a flame ionisation detector-Clarus 580 CPG/FID). 
Total organic carbon (TOC) analysis was performed by TOC Analyzer Formacs HT. The determination of phosphate, nitrate, and nitrite was carried out using a DR1900 colourimetric spectrophotometer at a wavelength of $860 \mathrm{~nm}$ using the different LCK reagents.

\subsection{Photocatalytic Degradation Measurement}

The photocatalytic degradation of oily wastewater was performed in a solar photoreactor located in the north of Algeria (latitude $36.39^{\circ}$; longitude $2.42^{\circ}$ at sea level), using natural sunlight radiation. A Pyranometer CMP 11 (Kipp \& Zonen, Delft, The Netherlands) with a spectral range between 285 and $2800 \mathrm{~nm}$ was used to measure the solar UV intensity. The reactor was developed at the Solar Equipment Development Unit (UDES) in Algeria. The capacity of the photoreactor is $1 \mathrm{~L}$ ( $38 \mathrm{~cm}$ length $\times 12 \mathrm{~cm}$ wide $\times 8.5 \mathrm{~cm}$ high). The photoreactor tank was fabricated from Pyrex glass, where the produced 8 wt. $\% \mathrm{TiO}_{2} / \mathrm{PVDF}-\mathrm{TrFE}$ nanocomposite membrane was placed at the bottom (Figure 1). The flow rate used to recirculate the oily water was $100.8 \mathrm{~L} \mathrm{~h}^{-1}$; in this way, it was possible to completely cover the nanocomposite membrane with the oily water. The photoreactor was wholly covered with glass to avoid evaporation during the photocatalytic experiments.
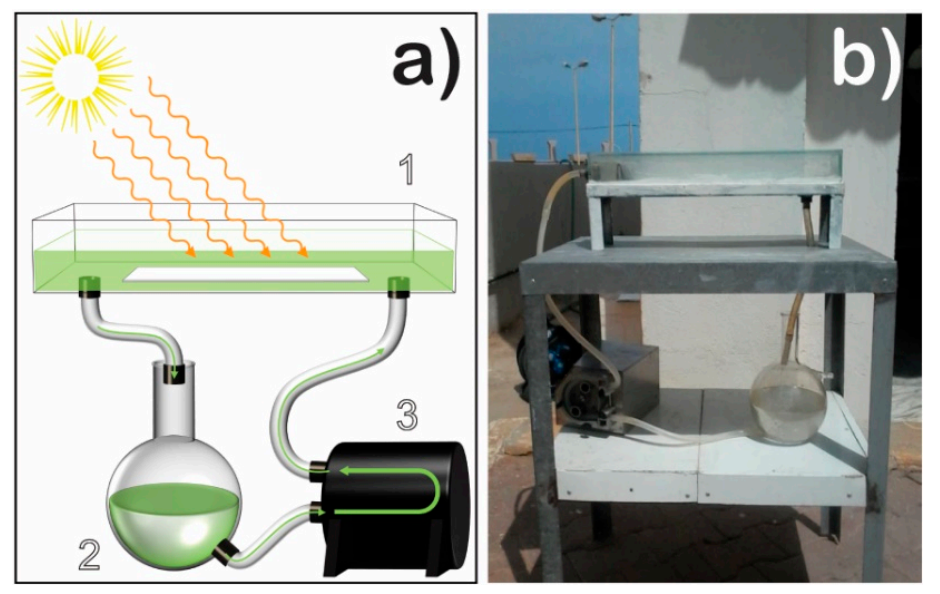

Figure 1. Representation of the solar photoreactor: (a) (1) photoreactor tank with the photocatalytic membrane at the bottom; (2) oily wastewater; (3) peristaltic pump; (b) a picture of the real photoreactor setup.

For the photocatalytic assays, one liter of oily wastewater solution was added to the photoreactor tank containing the membrane and exposed to solar illumination for seven hours, which is the period that assures a constant sunlight irradiance intensity during the experiment.

The degradation of oily wastewater was analyzed with a UV-visible spectrophotometer (Shimadzu-1800, Duisburg, Germany), and the peak at $262 \mathrm{~nm}$ was used to monitor oily wastewater absorbance over the irradiation time.

\section{Results and Discussion}

The nanoparticles and membrane characterizations are summarized in Figure 2. The crystalline structure of $\mathrm{TiO}_{2}$ nanoparticles was assessed by X-ray diffraction (XRD) and compared with the pattern diffractograms (Figure 2a). The diffractogram indicates intense reflexes at $2 \theta \approx 25.39^{\circ}(101), 37.13^{\circ}$ (103), 37.89 $(004), 38.65^{\circ}(112), 48.09^{\circ}(200), 53.99^{\circ}(105), 55.15^{\circ}(211)$, and $62.81^{\circ}$ (118), corresponding to the anatase phase (A), in agreement with the standard spectrum of anatase JCPDS 21-1272 [29-31] and JCPDS no.: $84-1286$ [32]. In the same sample there are also diffraction values at $2 \theta \approx 27.49^{\circ}(110)$, $36.15^{\circ}(101)$, and $56.65^{\circ}(220)$ that are in agreement with the standard spectrum of the rutile phase (R) JCPDS 88-1175 [32]. 

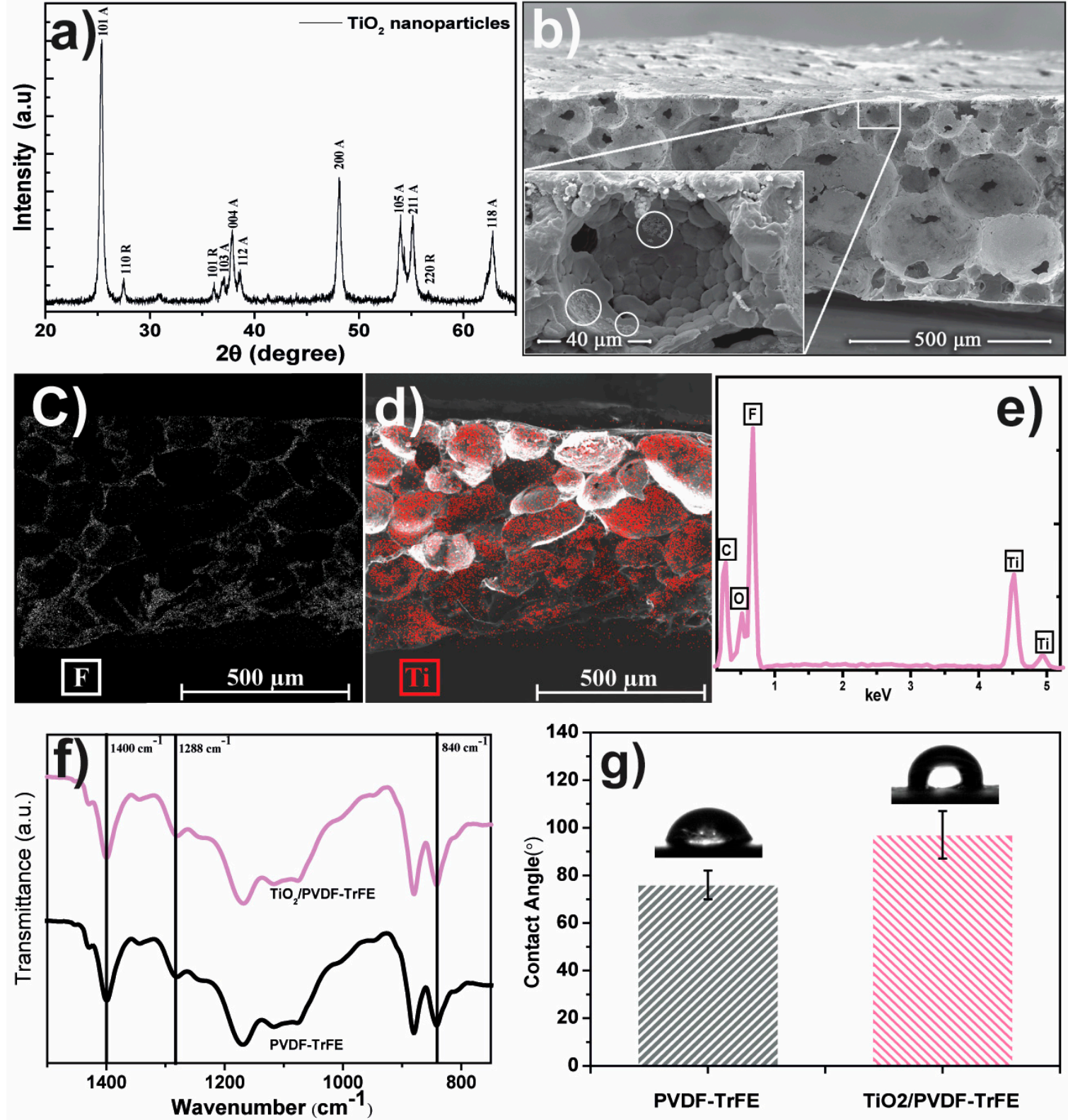

Figure 2. (a) $\mathrm{XRD}$ pattern of $\mathrm{TiO}_{2}$ nanoparticles and identification of the different crystal phases: Anatase (A), and rutile (R). (b) Cross-section SEM images of $8 \mathrm{wt} . \% \mathrm{TiO}_{2} /$ poly(vinylidene fluoride-trifluoro ethylene) (PVDF-TrFE) membranes with an inset showing a detail of an interconnected pores, with white circles for $\mathrm{TiO}_{2}$ nanoparticles and aggregates. SEM-EDX mapping image of the presence and distribution of $\mathrm{F}$ (fluorine) (c), and Ti (red) (d), in the PVDF-TrFE matrix and the EDX spectrum with the identification of the detected elements (e). FTIR spectra and contact angle of $8 \mathrm{wt} . \% \mathrm{TiO}_{2} / \mathrm{PVDF}-\mathrm{TrFE}$ nanocomposite membranes and pure polymer, $(\mathbf{f}, \mathbf{g})$ respectively.

SEM cross-section images, Figure $2 b$, shows the porous microstructure of the produced membrane with the characteristic interconnected (small pores inside the pores) spherical pores with an average diameter of $\approx 60 \mu \mathrm{m}$. Moreover, it is possible to identify $\mathrm{TiO}_{2}$ nanoparticles and agglomerates attached to the pore walls, signaled with circles in the inset of Figure $1 \mathrm{~b}-$ SEM surface micrographs are available as supplementary material (Figure S1). The EDX mapping, Figure 2c, d, allows confirmation of the homogeneous distribution of titanium (Ti) and fluorine (F), ascribed to the presence of $\mathrm{TiO}_{2}$ nanoparticles over the porous PVDF-TrFE matrix, respectively. The EDX spectrum confirms the presence of all elements present on the nanocomposite, Figure 2e. 
Infrared spectroscopy (FTIR) was used to determine the polymer phase and the possible chemical interaction between fillers and polymer matrix. In Figure 2c, the FTIR spectra of $8 \mathrm{wt} . \%$ $\mathrm{TiO}_{2} / \mathrm{P}(\mathrm{VDF}-\mathrm{TrFE})$ nanocomposite show a stable polymer structure concerning the pure polymer. Bands at 840,1288 and $1400 \mathrm{~cm}^{-1}$ show that the polymer crystallizes in the all-trans piezoelectric $\beta$-phase [27].

The polymer phase remains unchanged when $\mathrm{TiO}_{2}$ is present in the membranes, as already reported in Martins et al. [28]. In this way, filler content and type do not change the crystallization phase of the polymer, which crystallizes in the all-trans $\beta$-phase, and no chemical bonds are detected between polymer and fillers.

Contact angle measurements assessed the wettability of the membranes. Figure $2 \mathrm{~d}$ illustrates the contact angle for the pristine and the $8 \mathrm{wt} . \%$ of $\mathrm{TiO}_{2} / \mathrm{PVDF}-\mathrm{TrFE}$ samples, which present contact angles of $97^{\circ}$ and $76^{\circ}$, respectively. These results show the decrease of the contact angle with the incorporation of $\mathrm{TiO}_{2}$ nanoparticles on the polymer matrix, which in turn, allows for a higher interaction between the nanocatalyst and the oily water.

\subsection{Photocatalytic Degradation of Oily Wastewater}

\subsubsection{Effect of Initial Chemical Oxygen Demand}

Before the photocatalytic assays, controls were performed using oily wastewater without membrane (photolysis), and oily water with pristine PVDF-TrFE (adsorption), and in both cases the degradation was neglectable $(\approx 1 \%)$ - see results as supplementary material Figure S2. The original oily water, with a COD value of $51,758 \mathrm{mg} / \mathrm{L}$, was diluted with distilled water to obtain approximately half of the initial COD value $\approx 25,879 \mathrm{mg} / \mathrm{L}$. Afterwards, both samples were placed on the solar photoreactor to perform a degradation under the same conditions. The obtained results are shown in Table 1.

Table 1. Chemical oxygen demand (COD) values and respective removal rate (\%) for oily water after treatment in photoreactor with the $8 \% \mathrm{TiO}_{2} / \mathrm{P}(\mathrm{VDF}-\mathrm{TrFE})$ membrane after seven hours under sun irradiation.

\begin{tabular}{cc}
\hline COD (mg/L) & Removal Rate (\%) \\
\hline 51,758 & 49 \\
25,879 & 99.6 \\
\hline
\end{tabular}

This experiment has indicated an increased removal efficiency after diluting the initial oily water sample, with removal rates (R\%) of $49 \%$ and $99.6 \%$ for the COD values of 51,758 and $25,879 \mathrm{mg} / \mathrm{L}$, respectively. Such enhanced efficiency is related to turbidity reduction after dilution, from 425 NTU to 205 NTU. Decreasing turbidity favors the penetration of the sunlight radiation, which leads to oil removal rate improvement.

The $8 \mathrm{wt} . \% \mathrm{TiO}_{2} / \mathrm{P}(\mathrm{VDF}-\mathrm{TrFE})$ membranes were used to assess the degradation of oily wastewater in a solar photoreactor. Figure 3a shows the shift of transparency of the oily water after $7 \mathrm{~h}$ under sunlight radiation.

The visual inspection indicates that the oily wastewater pollutants were degraded as colorless water was obtained after the seven hours. As confirmation, Figure 3b shows the absorption spectra of oily water samples before and after the photocatalytic treatment. The maximum absorption band is located at $262 \mathrm{~nm}$. The decrease of the absorption peak at $262 \mathrm{~nm}$ confirms the photodegradation of oily water. 

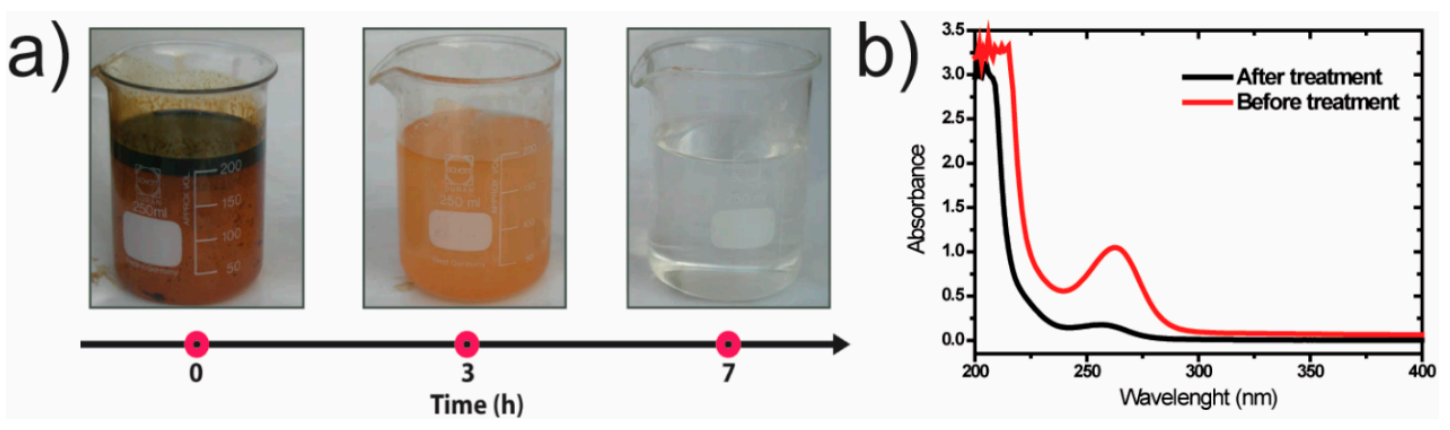

Figure 3. (a) The appearance of the oily wastewater along with the treatment with the 8 wt. $\%$ $\mathrm{TiO}_{2} / \mathrm{P}(\mathrm{VDF}-\mathrm{TrFE})$ nanocomposite membrane in the solar photoreactor; (b) UV-vis absorption spectrum before and after the photocatalytic treatment of oily water after $7 \mathrm{~h}$ under sun irradiation/

\subsubsection{Effect of Oily Water $\mathrm{pH}$}

In a different set of assays, the $\mathrm{pH}$ influence on the oily water degradation process was assessed; thus, three oily water solutions were prepared, and the initial $\mathrm{pH}$ was adjusted either with sulfuric acid $\left(\mathrm{H}_{2} \mathrm{SO}_{4}\right)$ or sodium hydroxide $(\mathrm{NaOH})$ solutions.

The results obtained indicate that the photocatalytic degradation is strongly related to the initial $\mathrm{pH}$ of the oily water solution. The highest removal efficiency, approximately $80 \%$, was obtained at the initial $\mathrm{pH}$ of 5.5. Moreover, the results indicate that acid or basic solutions reduce the photocatalytic efficiency below $40 \%$ of COD removal.

\subsubsection{Wastewater Parameters}

Additionally, other parameters that were measured for oily wastewater, before and after the treatment, are shown in Table 2.

Table 2. Main parameters of the wastewater before and after the proposed treatment.

\begin{tabular}{|c|c|c|c|c|}
\hline Parameter & Before Treatment & After Treatment & Removed (\%) & Limit Values $^{1}$ \\
\hline $\mathrm{pH}$ & 4.3 & 5.3 & - & $6.5-8.5$ \\
\hline Temperature $\left({ }^{\circ} \mathrm{C}\right)$ & 21.1 & 19.1 & 9.47 & 30 \\
\hline Turbidity (NTU) & 205 & 7 & 96.58 & 10 \\
\hline $\operatorname{COD}\left(\mathrm{mg} \mathrm{L}^{-1}\right)$ & 25879 & 105 & 99.59 & 120 \\
\hline Conductivity $\left(\mathrm{ms} \mathrm{cm}^{-1}\right)$ & 236 & 167.4 & 29.06 & 2 \\
\hline Chloride $\left(\mathrm{mg} \mathrm{L}^{-1}\right)$ & 1047.4 & 804.2 & 25.82 & 500 \\
\hline Suspended solids $\left(\mathrm{mg} \mathrm{L}^{-1}\right)$ & 73.9 & 17.9 & 75.77 & 35 \\
\hline Total hydrocarbons $\left(\mathrm{mg} \mathrm{L}^{-1}\right)$ & 48.69 & 0.1492 & 99.69 & 10 \\
\hline $\operatorname{TOC}\left(\mathrm{mg} \mathrm{L}^{-1}\right)$ & 872 & 19.81 & 97.72 & 20 \\
\hline Nitrate $\left(\mathrm{mg} \mathrm{L}^{-1}\right)$ & 440 & 137 & 68.86 & 50 \\
\hline Nitrite $\left(\mathrm{mg} \mathrm{L}^{-1}\right)$ & 0.99 & 0 & 100 & 1 \\
\hline Phosphate $\left(\mathrm{mg} \mathrm{L}^{-1}\right)$ & 0.17 & 0 & 100 & 2 \\
\hline
\end{tabular}

${ }^{1}$ Executive Decree No. 06-141 of 20 Rabie El Aouel 1427 corresponding to 19 April 2006, defining the limit values for discharges of industrial liquid effluents of Algeria.

The results obtained after seven hours of sunlight irradiation of the $8 \mathrm{wt} . \% \mathrm{TiO}_{2} / \mathrm{PVDF}-\mathrm{TrFE}$ membrane in contact with the oily wastewater in the photoreactor show a COD removal around $99 \%$. The suspended solids concentration was largely removed, and only $24 \%$ of the solids remained in the solution. There was also a significant decrease in the conductivity that is generally associated with nitrogen removal (in agreement with measured parameters) [33]. The total hydrocarbons (HC) were completely removed $(>99 \%)$, which indicates the efficient removal of the oil in the wastewater. Furthermore, there was a reduction in all the assessed parameters, especially nitrate reduction, and all the final values were below the legal limits. It is also relevant to highlight that these measurements confirm that the highest COD removals are not obtained in extremely acidic or basic $\mathrm{pH}^{\prime} \mathrm{s}$, as confirmed in the previous section (Figure 4). 


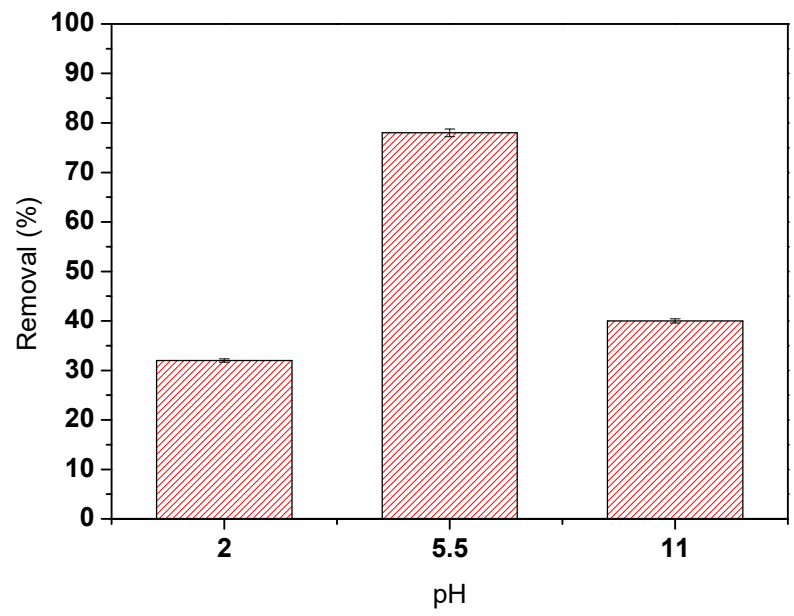

Figure 4. The influence of the initial $\mathrm{pH}$ on oily water photocatalytic degradation.

The reusability of the $\mathrm{TiO}_{2} / \mathrm{PVDF}-\mathrm{TrFE}$ was formerly proved in [24], which also contributes to making this material suitable for applications.

The disappearance of peaks in the chromatogram obtained by gas chromatographic analysis (GC-FID) confirms the efficient removal of total hydrocarbons, Figure 5.

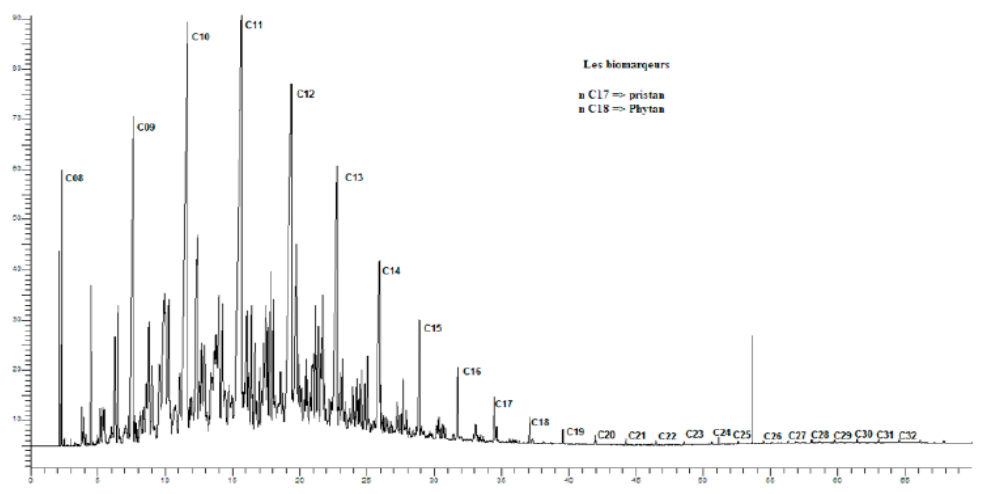

(a)

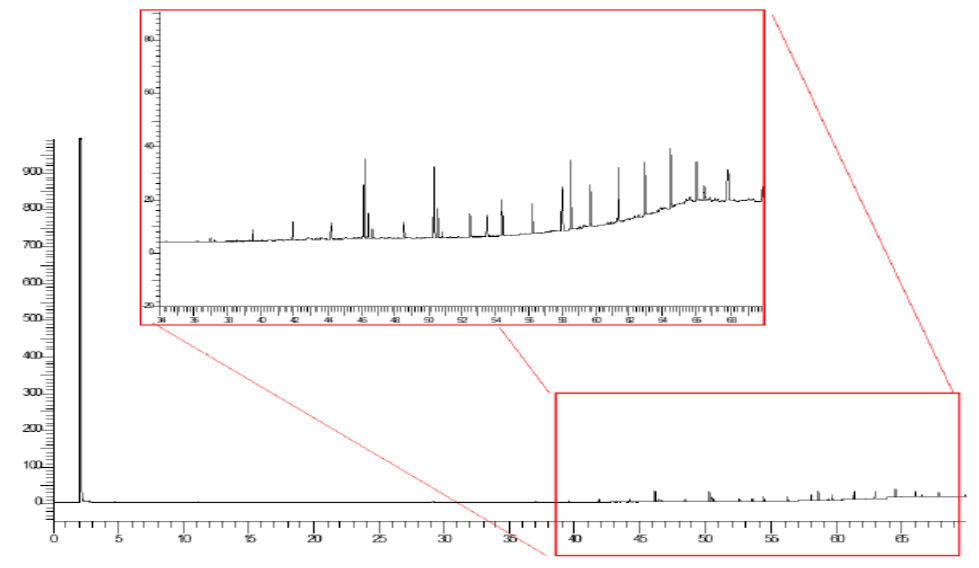

(b)

Figure 5. Chromatogram corresponding to the sample before (a) and after (b) the photocatalytic degradation in seven hours of sunlight irradiation. 
The majority of the organic compounds present in the sample before treatment (Figure 5a) belong to the alkanes family (C10-C30), which is in good agreement with similar works [6,34]. After the photocatalytic treatment, the presence of all these compounds was reduced, Figure $5 \mathrm{~b}$. The long-chain hydrocarbons were degraded by photocatalytic activity into smaller organic compounds.

The results show that by using the $8 \mathrm{wt} . \% \mathrm{TiO}_{2} / \mathrm{PVDF}-\mathrm{TrFE}$ nanocomposite membrane, it is possible to treat oily industrial wastewater, such as that from the central region of Hassi Rmel Algeria, using the sun as a source of energy.

\section{Conclusions}

This work reports the preparation of a $\mathrm{TiO}_{2} / \mathrm{P}(\mathrm{VDF}-\mathrm{TrFE})$ nanocomposite by solvent casting and its photocatalytic application in the remediation of oily wastewater. The characterization of the samples shows a homogeneous distribution of the $\mathrm{TiO}_{2}$ nanoparticles over the pores with an interconnected structure. FTIR and contact angle results prove the chemical stability and the unchanged hydrophobic nature of the nanocomposite membrane. The photocatalytic assays allowed complete removal of the COD (99.6\%), total hydrocarbons (99.6\%), and phosphates (100\%), as well as a significant reduction of the suspended solids $(76 \%)$, turbidity $(97 \%)$, TOC $(98 \%)$, nitrates $(69 \%)$, and chloride $(26 \%)$ from oily wastewater. The $\mathrm{pH}$ range that allows better removal efficiencies ranges between 4 and 5.5. After seven hours of sunlight exposure, colourless oily wastewater was obtained. Thus, the fabricated membranes combine an inexpensive nanocatalyst and UV radiation source (available in sunlight radiation) to efficiently remediate oily water. Therefore, the $\mathrm{TiO}_{2} / \mathrm{P}(\mathrm{VDF}-\mathrm{TrFE})$ nanocomposite membranes proved to possess a proper morphology and chemical stability envisaging oily wastewater remediation and related applications.

Supplementary Materials: The following are available online at http://www.mdpi.com/2073-4360/12/1/42/s1, Figure S1: Surface SEM image of a TiO2/PVDF-TrFE nanocomposite. Figure S2: Photocatalytic controls performed with just oily water, PVDF-TrFE, and the TiO2/PVDF-TrFE, under the same conditions.

Author Contributions: Conceptualization, D.Z., L.A., S.L.-M.; methodology, D.Z., L.A., H.S., and P.M.M.; validation, H.S., P.M.M., D.Z., and S.L.-M.; formal analysis, P.M.M., S.L.-M.; investigation, D.Z., L.A., H.S., P.M.M.; resources, D.Z., and S.L.-M.; writing-original draft preparation, D.Z., L.A., H.S.; writing-P.M.M. and S.L.-M.; supervision, S.L.-M. All authors have read and agreed to the published version of the manuscript.

Funding: This work was supported by the Solar Equipment Development Unit (UDES) Algeria. This work was also supported by the Portuguese Foundation for Science and Technology (FCT) in the framework of the strategic project UID/FIS/04650/2013 by FEDER funds through the COMPETE 2020-Programa Operacional Competitividade e Internacionalização (POCI) with the reference project POCI-01-0145-FEDER-006941, and project PTDC/CTM-ENE/5387/2014.

Acknowledgments: P.M. Martins and H. Salazar thanks the FCT for the grants SFRH/ BD/ 98616/2013 and SFRH/BD/122373/2016. The authors acknowledge funding from the Basque Government Industry Department under the ELKARTEK and HAZITEK programs and the Spanish Ministry of Economy and Competitiveness (MINECO) through the project MAT2016-76039-C4-3-R(AEI/FEDER, UE) (including the FEDER financial support).

Conflicts of Interest: The authors declare no conflict of interest.

\section{References}

1. Rusli, U.N.; Alias, N.H.; Shahruddin, M.Z.; Othman, N.H. Photocatalytic Degradation of Oil using Polyvinylidene Fluoride/Titanium Dioxide Composite Membrane for Oily Wastewater Treatment. In Proceedings of the MATEC Web Conference, Shah Alam, Selangor, Malaysia, 2 August 2016; Volume 69, p. 05003.

2. Sun, S.; Xiao, Q.R.; Zhou, X.; Wei, Y.Y.; Shi, L.; Jiang, Y. A bio-based environment-friendly membrane with facile preparation process for oil-water separation. Colloids Surf. Physicochem. Eng. Asp. 2018, 559, 18-22. [CrossRef]

3. Stack, L.J.; Carney, P.A.; Malone, H.B.; Wessels, T.K. Factors influencing the ultrasonic separation of oil-in-water emulsions. Ultrason. Sonochem. 2005, 12, 153-160. [CrossRef] [PubMed]

4. Soares, S.F.; Rodrigues, M.I.; Trindade, T.; Daniel-da-Silva, A.L. Chitosan-silica hybrid nanosorbents for oil removal from water. Colloids Surf. Physicochem. Eng. Asp. 2017, 532, 305-313. [CrossRef] 
5. Cañizares, P.; Martínez, F.; Jiménez, C.; Sáez, C.; Rodrigo, M.A. Coagulation and electrocoagulation of oil-in-water emulsions. J. Hazard. Mater. 2008, 151, 44-51. [CrossRef]

6. Ong, C.S.; Lau, W.J.; Goh, P.S.; Ng, B.C.; Ismail, A.F. Investigation of submerged membrane photocatalytic reactor (sMPR) operating parameters during oily wastewater treatment process. Desalination 2014, 353, 48-56. [CrossRef]

7. Sun, Y.; Zong, Y.; Yang, N.; Zhang, N.; Jiang, B.; Zhang, L.; Xiao, X. Surface hydrophilic modification of PVDF membranes based on tannin and zwitterionic substance towards effective oil-in-water emulsion separation. Sep. Purif. Technol. 2020, 234, 116015. [CrossRef]

8. Zhou, W.; Fang, Y.; Li, P.; Yan, L.; Fan, X.; Wang, Z.; Zhang, W.; Liu, H. Ampholytic Chitosan/Alginate Composite Nanofibrous Membranes with Super Anti-Crude Oil-Fouling Behavior and Multifunctional Oil/Water Separation Properties. ACS Sustain. Chem. Eng. 2019, 7, 15463-15470. [CrossRef]

9. Zhang, W.; Li, X.; Qu, R.; Liu, Y.; Weia, Y.; Feng, L. Janus membrane decorated: Via a versatile immersion-spray route: Controllable stabilized oil/water emulsion separation satisfying industrial emission and purification criteria. J. Mater. Chem. A 2019, 7, 4941-4949. [CrossRef]

10. Zhang, C.; Zhang, Y.; Xiao, X.; Liu, G.; Xu, Z.; Wang, B.; Yu, C.; Ras, R.H.A.; Jiang, L. Efficient separation of immiscible oil/water mixtures using a perforated lotus leaf. Green Chem. 2019, 21, 6579-6584. [CrossRef]

11. Zhang, B.; Yu, S.; Zhu, Y.; Shen, Y.; Gao, X.; Shi, W.; Tay, J.H. Adsorption mechanisms of crude oil onto polytetrafluoroethylene membrane: Kinetics and isotherm, and strategies for adsorption fouling control. Sep. Purif. Technol. 2020, 235, 116212. [CrossRef]

12. Yagoub, H.; Zhu, L.; Shibraen, M.H.M.A.; Altam, A.A.; Babiker, D.M.D.; Rehan, K.; Mukwaya, V.; Xu, J.; Yang, S. Manipulating the surface wettability of polysaccharide based complex membrane for oil/water separation. Carbohydr. Polym. 2019, 225, 115231. [CrossRef] [PubMed]

13. Yang, Y.; Liang, W.; Wang, C.; Sun, H.; Zhang, J.; Yu, Y.; Dong, W.; Zhu, Z.; Li, A. Fabrication of palygorskite coated membrane for multifunctional oil-in-water emulsions separation. Appl. Clay Sci. 2019, 182, 105295. [CrossRef]

14. Saththasivam, J.; Wubulikasimu, Y.; Ogunbiyi, O.; Liu, Z. Fast and efficient separation of oil/saltwater emulsions with anti-fouling $\mathrm{ZnO}$ microsphere/carbon nanotube membranes. J. Water Process Eng. 2019, 32, 100901. [CrossRef]

15. Wang, Z.X.; Lau, C.H.; Zhang, N.Q.; Bai, Y.P.; Shao, L. Mussel-inspired tailoring of membrane wettability for harsh water treatment. J. Mater. Chem. A 2015, 3, 2650-2657. [CrossRef]

16. Zhang, X.; Wang, Y.; Liu, Y.; Xu, J.; Han, Y.; Xu, X. Preparation, performances of PVDF/ZnO hybrid membranes and their applications in the removal of copper ions. Appl. Surf. Sci. 2014, 316, 333-340. [CrossRef]

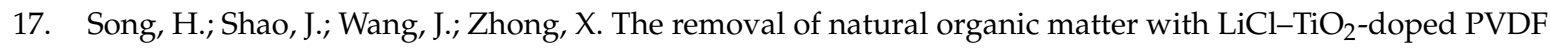
membranes by integration of ultrafiltration with photocatalysis. Desalination 2014, 344, 412-421. [CrossRef]

18. Zhang, M.; Zhang, L.; Cheng, L.-H.; Xu, K.; Xu, Q.-P.; Chen, H.-L.; Lai, J.-Y.; Tung, K.-L. Extracorporeal endotoxin removal by novel l-serine grafted PVDF membrane modules. J. Membr. Sci. 2012, 405, 104-112. [CrossRef]

19. Gao, L.; Alberto, M.; Gorgojo, P.; Szekely, G.; Budd, P.M. High-flux PIM-1/PVDF thin film composite membranes for 1-butanol/water pervaporation. J. Membr. Sci. 2017, 529, 207-214. [CrossRef]

20. Ramaiah, K.P.; Satyasri, D.; Sridhar, S.; Krishnaiah, A. Removal of hazardous chlorinated VOCs from aqueous solutions using novel ZSM-5 loaded PDMS/PVDF composite membrane consisting of three hydrophobic layers. J. Hazard. Mater. 2013, 261, 362-371. [CrossRef]

21. Zuo, X.; Shi, W.; Tian, Z.; Yu, S.; Wang, S.; He, J. Desalination of water with a high degree of mineralization using $\mathrm{SiO}_{2} / \mathrm{PVDF}$ membranes. Desalination 2013, 311, 150-155. [CrossRef]

22. Leaper, S.; Abdel-Karim, A.; Faki, B.; Luque-Alled, J.M.; Alberto, M.; Vijayaraghavan, A.; Holmes, S.M.; Szekely, G.; Badawy, M.I.; Shokri, N.; et al. Flux-enhanced PVDF mixed matrix membranes incorporating APTS-functionalized graphene oxide for membrane distillation. J. Membr. Sci. 2018, 554, 309-323. [CrossRef]

23. Salazar, H.; Nunes-Pereira, J.; Correia, D.M.; Cardoso, V.F.; Gonçalves, R.; Martins, P.M.; Ferdov, S.; Martins, M.D.; Botelho, G.; Lanceros-Méndez, S. Poly(vinylidene fluoride-hexafluoropropylene)/bayerite composite membranes for efficient arsenic removal from water. Mater. Chem. Phys. 2016, 183, 430-438. [CrossRef] 
24. Aoudjit, L.; Martins, P.M.; Madjene, F.; Petrovykh, D.Y.; Lanceros-Mendez, S. Photocatalytic reusable membranes for the effective degradation of tartrazine with a solar photoreactor. J. Hazard. Mater. 2018, 344, 408-416. [CrossRef] [PubMed]

25. Martins, P.M.; Miranda, R.; Marques, J.; Tavares, C.J.; Botelho, G.; Lanceros-Méndez, S. Comparative efficiency of $\mathrm{TiO}_{2}$ nanoparticles in suspension vs. immobilization into P(VDF-TrFE) porous membranes. RSC Adv. 2016, 6, 12708-12716. [CrossRef]

26. Zhang, W.; Ding, L.; Luo, J.; Jaffrin, M.Y.; Tang, B. Membrane fouling in photocatalytic membrane reactors (PMRs) for water and wastewater treatment: A critical review. Chem. Eng. J. 2016, 302, 446-458. [CrossRef]

27. Ribeiro, C.; Costa, C.M.; Correia, D.M.; Nunes-Pereira, J.; Oliveira, J.; Martins, P.; Gonçalves, R.; Cardoso, V.F.; Lanceros-Méndez, S. Electroactive poly(vinylidene fluoride)-based structures for advanced applications. Nat. Protoc. 2018, 13, 681. [CrossRef] [PubMed]

28. Cardoso, V.F.; Correia, D.M.; Ribeiro, C.; Fernandes, M.M.; Lanceros-Méndez, S. Fluorinated polymers as smart materials for advanced biomedical applications. Polymers 2018, 10, 161. [CrossRef]

29. Ye, M.; Zorba, S.; He, L.; Hu, Y.; Maxwell, R.T.; Farah, C.; Zhanga, Q.; Yin, Y. Self-assembly of superparamagnetic magnetite particles into peapod-like structures and their application in optical modulation. J. Mater. Chem. 2010, 20, 7965-7969. [CrossRef]

30. Wu, S.-H.; Wu, J.L.; Jia, S.Y.; Chang, Q.W.; Ren, H.; Liu, Y. Cobalt (II) phthalocyanine-sensitized hollow $\mathrm{Fe}_{3} \mathrm{O}_{4} @ \mathrm{SiO}_{2} @ \mathrm{TiO}_{2}$ hierarchical nanostructures: Fabrication and enhanced photocatalytic properties. Appl. Surf. Sci. 2013, 287, 389-396. [CrossRef]

31. Li, X.; Liu, D.; Song, S.; Zhang, H. $\mathrm{Fe}_{3} \mathrm{O}_{4} @ \mathrm{SiO}_{2} @ \mathrm{TiO}_{2} @$ Pt Hierarchical Core-Shell Microspheres: Controlled Synthesis, Enhanced Degradation System, and Rapid Magnetic Separation to Recycle. Cryst. Growth Des. 2014, 14, 5506-5511. [CrossRef]

32. Thamaphat, K.; Limsuwan, P.; Ngotawornchai, B. Phase characterization of $\mathrm{TiO}_{2}$ powder by XRD and TEM. Kasetsart J. Nat. Sci. 2008, 42, 357-361.

33. Levlin, E. Conductivity measurements for controlling municipal waste-water treatment. In Proceedings of the Polish-Swedish-Ukrainian Seminar, Ustron, Poland, 23-24 November 2007.

34. Li, G.; An, T.; Chen, J.; Sheng, G.; Fu, J.; Chen, F.; Zhang, S.; Zhao, H. Photoelectrocatalytic decontamination of oilfield produced wastewater containing refractory organic pollutants in the presence of high concentration of chloride ions. J. Hazard. Mater. 2006, 138, 392-400. [CrossRef] [PubMed] 